\title{
Existence and Blow-up Properties of Solutions for A Non-Newtonian Filtration Equation
}

\author{
Zhongyang Qiao \\ The Normal School of Beihua University \\ Jilin City PR China
}

\begin{abstract}
In this paper, a non-Newtonian filtration equation with nonlinear boundary condition and a localized source is considered. The existence and blow-up properties of solutions are given under some conditions.
\end{abstract}

Keywords-existence; blow-up; non-newtonian filtration equation; localized source

\section{INTRODUCTION}

In this paper, we consider the positive solutions to the following non-Newtonian filtration equation

$$
\begin{aligned}
& u_{t}=\operatorname{div}\left(|\nabla u|^{m-2} \nabla u\right)+c u^{p}\left(x_{0}, t\right) \int_{\Omega} u^{q} d x \\
& x \in \Omega, t>0 \text {, } \\
& u(x, t)=\int_{\Omega} k(x, y) u(y, t) d y, \quad x \in \partial \Omega, t>0, \\
& u(x, 0)=u_{0}(x), \quad x \in \Omega,
\end{aligned}
$$

where $m>2, p>0, q \geq 0$ are constants and $c>0, \Omega$ is a bounded domain in $R^{N}(N \geq 1)$ with smooth boundary $\partial \Omega . k(x, y)$ is a nonnegative continuous function for $(x, y) \in \partial \Omega \times \bar{\Omega}$, while $u_{0}(x)$ is a positive continuous function.

There have been many literature which consider properties of solutions to partial differential equations with nonlinear boundary conditions. However, there are some important phenomena formulated into parabolic equations which are coupled with nonlinear boundary conditions in mathematical modelling such as thermoelasticity theory (see [1]-[4]. In this case, the solution $u(x, t)$ describes entropy per volume of material.

Recently, A. Gladkow, K. Ik Kim in [2] studied the following semilinear heat equation problem with nonlinear boundary condition and local reaction term

$$
\left\{\begin{array}{l}
u_{t}=\Delta u+c(x, t) u^{p}, \quad x \in \Omega, t>0, \\
u(x, t)=\int_{\Omega} k(x, y) u^{l}(y, t) d y, \quad x \in \partial \Omega, t>0, \\
\quad u(x, 0)=u_{0}(x), \quad x \in \Omega,
\end{array}\right.
$$

where $p>1, l>0$ are constants, $u_{0}(x)$ and $k(x, y)$ satisfy the same assumptions in Problem (1).

Non-Newtonian filtration equations and the equations of its type with source or local boundary conditions have been extensively studied (see [5, 6]). However, to our best knowledge, there seem to be few articles on non-Newtonian filtration equations with both nonlocal boundary conditions and localized sources. The main purpose of this paper is establish the global existence and finite time blowup of the solutions.

\section{COMPARISON PRINCIPLE}

In this section, we give the comparison principle for Problem (1).

Let

$$
Q_{T}=\Omega \times(0, T)
$$

and

$\Gamma_{T}=\partial \Omega \times(0, T) \bigcup \bar{\Omega} \times\{t=0\}$. We begin with the definition of subsolution and supersolution to Problem (1).

Definition 2.1 A function $\underline{u}(x, t)$ is called a subsolution to Problem (1) on $Q_{T}$, if $\underline{u} \in C^{2,1}\left(Q_{T}\right) \cap C\left(\bar{Q}_{T}\right)$ satisfies

$$
\left\{\begin{array}{l}
\underline{u}_{t} \leq \operatorname{div}\left(\mid \nabla \underline{u}^{m-2} \nabla \underline{u}\right)+c \underline{u}^{p}\left(x_{0}, t\right) \int_{\Omega} \underline{u}^{q} d x, \\
x \in \Omega, t>0, \\
\underline{u}(x, t) \leq \int_{\Omega} k(x, y) \underline{u}(y, t) d y, \quad x \in \partial \Omega, t>0, \\
\underline{u}(x, 0) \leq u_{0}(x), \quad x \in \Omega .
\end{array}\right.
$$

A supersolution is defined in similar way with each inequality reversed.

Lemma 2.1 Let $u$ and $v$ be a nonnegative subsolution and a supersolution to $(1)$, respectively, with $u(x, 0) \leq v(x, 0)$ for $x \in \bar{\Omega}$. Then $u \leq v$ in $Q_{T}$, if either $v \geq \delta>0$ or $u \geq \delta>0$ holds. 
The technique for proving the comparison principle is quite standard. Readers may refer to [4] and we omit it here.

To obtain the blow-up rate estimates, we need the following positivity lemma, whose proof is much the same as that in [5].

Lemma 2.2 Suppose that $w \in C\left(Q_{T} \cup \Gamma_{T}\right) \cap C^{2,1}\left(Q_{T}\right)$ satisfies

$$
\left\{\begin{array}{r}
w_{t}-d(x, t) \Delta w \geq c_{1}(x, t) w+c_{2}(x, t) w\left(x_{0}, t\right) \int_{\Omega} w^{q} d x \\
(x, t) \in Q_{T} \\
w(x, t) \geq \int_{\Omega} c_{3}(x, y) w(y, t) d y,(x, t) \in \partial \Omega \times(0, T)
\end{array}\right.
$$

where $c_{i}(x, t)(i=1,2,3)$ is a bounded function in its domain, $\quad d(x, t)>0, c_{2}(x, t) \geq 0,(x, t) \in Q_{T} \quad$, $c_{3}(x, y) \geq 0, \quad(x, y) \in \partial \Omega \times \Omega$ and is not identically zero. Then

$w(x, 0)>0$ for $x \in \bar{\Omega}$ implies $w(x, t)>0,(x, t) \in Q_{T}$. Moreover, if $c_{3}(x, y) \equiv 0 \quad$ or $\quad$ if $c_{3}(x, y) \geq 0$ and $\int_{\Omega} c_{3}(x, y) d y \leq 1, x \in \partial \Omega$, then $w(x, 0) \geq 0$ for $x \in \bar{\Omega}$ implies $w(x, t) \geq 0,(x, t) \in Q_{T}$.

\section{GLOBAL EXISTENCE AND BLOW-UP}

Theorem 3.1 Let $\int_{\Omega} k(x, y) d y=1$. Then every solution of Problem (1) exists globally for $p+q \leq 1$, while it blows up in finite time for $p+q>1$.

Proof Similar to the paper [3], we also construct a supersolution to Problem (1) on $Q_{T}$ when $p+q \leq 1$. Let $T$ be any positive number and $v(t)=\eta e^{c t}$. It is not difficult to verify that $v(t)$ is a supersolution of Problem (1) if $v(0)=\eta$ $\geq\left\{\max _{\bar{\Omega}} u_{0}(x), 1\right\}$ under the assumptions $p+q \leq 1$ and $\int_{\Omega} k(x, y) d y=1$. Since $v(t) \geq \eta$, we obtain $u(x, t) \leq v(t)$ by using the comparison principle, and hence $u(x, t)$ exists globally.

When $p+q>1$, we study the following ordinary differential equation

$$
v^{\prime}(t)=c_{0} v^{p+q}(t), \quad v(0)=v_{0}
$$

where $0<v_{0} \leq \min _{\bar{\Omega}} u_{0}(x), c_{0}=c|\Omega|,|\Omega|$ is the measure of $\Omega$. It is easy to see that $v(t)$ is a subsolution of Problem (1). It is known to us that the solution to (2) blows up in finite time. By using the comparison principle again, we obtain that the solution to (1) blows up in finite time.

Theorem 3.2 Let $\int_{\Omega} k(x, y) d y>1$. Then the solution to (1) blows up in finite time provided that $p+q>1$.

Proof. It is easy to verify that the solution for (2) is also a sub-solution to (1) under the conditions given in Theorem 3.2. So the solution of Problem (1) blows up in finite time.

Lemma 3.3[4] Let $\psi(x)$ be the unique positive solution of the following elliptical problem:

$$
\left\{\begin{array}{l}
-\operatorname{div}\left(|\nabla \psi|^{m-2} \nabla \psi\right)=a, x \in \Omega \\
\psi(x)=0, x \in \partial \Omega
\end{array}\right.
$$

Then we have $\psi(x)>0$, in $\Omega, \frac{\partial \psi}{\partial \eta}<0$ on the boundary $\partial \Omega$, and there exists a positive constant $A$ such that $A=\sup _{x \in \bar{\Omega}} \psi(x)<+\infty$.

Theorem 3.4 Assume that $\int_{\Omega} k(x, y) d y<\frac{1}{2}$.

(i) If $p+q+1<m$, then the solution of Problem (1) exists globally;

(ii) If $p+q+1=m$, then the solution of Problem (1) exists globally if $c$ is sufficiently small;

(iii) If $p+q+1>m$, then the solution of Problem (1) exists globally provided that $u_{0}(x)$ or $c$ is small, while $p>m>1, u(x, t)$ blows up in finite time if $u_{0}(x)$ is large enough.

Proof. Let $\phi(x)$ be the unique positive classical solution to the linear elliptic problem

$$
\left\{\begin{array}{l}
-\operatorname{div}\left(|\nabla \phi(x)|^{m-2} \nabla \phi(x)\right)=\varepsilon_{0}, x \in \Omega, \\
\phi(x)=0, \quad x \in \partial \Omega .
\end{array}\right.
$$

In view of lemma 3.3, we get $\phi(x)>0$, in $\Omega, \frac{\partial \phi}{\partial \eta}<0$ on $\partial \Omega$, and there exists a positive constant $A$ such that $A=\sup _{x \in \bar{\Omega}} \phi(x)<+\infty$. Define a function $w(x)$ as follows:

$$
w(x)=M\left(1+\frac{\phi(x)}{A}\right)
$$


where $M \geq 1$ is a constant to be determined later. Then, we have

$$
\begin{aligned}
& w_{t}-\operatorname{div}\left(|\nabla w|^{m-2} \nabla w\right)-c w^{p}\left(x_{0}, t\right) \int_{\Omega} w^{q} d x \\
&=\left(\frac{M}{A}\right)^{m-1} \varepsilon_{0}-c M^{p+q}\left(1+\frac{M}{A}\right)^{p}\left(1+\frac{M}{A}\right)^{q}|\Omega| \\
& \geq\left(\frac{M}{A}\right)^{m-1} \varepsilon_{0}-c M^{p+q}|\Omega| .
\end{aligned}
$$

(i) In the case of $p+q+1<m$, we have

$$
\begin{aligned}
\left.w\right|_{\partial \Omega} & =M \geq 2 M\left(\int_{\Omega} k(x, y) d y\right) \\
& \geq \int_{\Omega} k(x, y) M\left(1+\frac{\phi(x)}{A}\right) d y \\
& =\int_{\Omega} k(x, y) w(x) d y .
\end{aligned}
$$

Where we use the assumpution $\int_{\Omega} k(x, y) d y<\frac{1}{2}$.

Combining (4) with (5), we know that if we take

$$
M=\max \left\{\left(\frac{c|\Omega|}{\varepsilon_{0}} A^{m-1}\right)^{\frac{1}{m-1-p-q}}, \max _{\bar{\Omega}} u_{0}(x), 1\right\},
$$

then $w(x)$ defined as in (3) is a supersolution to (1) and $w(x) \geq M$. By using the comparison principle, we get $u(x, t) \leq w(x)$, and hence $u(x, t)$ exists globally.

(ii) In the case of $p+q+1=m$, we first choose $M>1$ large enough and

$$
u_{0}(x) \leq M\left(1+\frac{\phi(x)}{A}\right) .
$$

Set $c_{1}=\varepsilon_{0} A^{1-m}|\Omega|^{-1}$. Then it is easy to verify that $w(x)$ is a supersolution to Problem (1) provided $c_{1}>c$. Thus we know that $u(x, t)$ exists globally by using the comparison principle again.

(iii) In the case of $p+q+1>m$, there exist both global and blow-up solutions depending on the initial data and the coefficient $c$. For the global existence part, the proof is similar to that of (i) and (ii). For any given initial datum $u_{0}$, there exists a suitable large constant $M>0$ such that $u_{0} \leq M$.

For such a fixed $M$, set

$$
c_{2}=M^{m-p-q-1}|\Omega|^{-1} \varepsilon_{0} A^{1-m} .
$$

Then we know that $w(x)$ is a supersolution to Problem (1) if $c \leq c_{2}$. Again by using the comparison principle, we obtain the global existence of $u(x, t)$.

In according to prove the blow-up result, we consider a subsolution to Problem (1) in the self-similar form

$$
v(x, t)=\frac{1}{(T-t)^{l}} \omega\left(\frac{\left|x-x_{0}\right|}{(T-t)^{\sigma}}\right)
$$

with

$$
\omega(r)= \begin{cases}1+\frac{L}{2}-\frac{r^{2}}{2 L}, & 0 \leq r \leq R, \\ 0, & r>R,\end{cases}
$$

here $R, l, \sigma>0$ and $0<T<1$ is to be determined later. Clearly, $\quad 0 \leq \omega(r) \leq 1+\frac{L}{2}$ and $\omega^{\prime}(r) \leq 0$.

Note that

$$
\operatorname{supp} v(\cdot, t)=\overline{B\left(x_{0}, R(T-t)^{\sigma}\right)} \subset \overline{B\left(x_{0}, R T^{\sigma}\right)} \subset \Omega,
$$

for sufficiently small $T>0$. Obviously, $v(x, t)$ becomes unbounded as $t \rightarrow T^{-}$at the point $x=x_{0}$. Letting

$$
r=\frac{x-x_{0}}{(T-t)^{\sigma}},
$$

and calculating directly we obtain

Case 1: If $0 \leq r \leq A$, we have $1 \leq \omega(r) \leq 1+\frac{A}{2}$, and we obtain

$$
\begin{gathered}
v_{t}-\operatorname{div}\left(|\nabla v|^{m-2} \nabla v\right)-c v^{p}\left(x_{0}, t\right) \int_{\Omega} v^{q}(x, t) d x \\
\leq \frac{l\left(1+\frac{A}{2}\right)}{(T-t)^{l+1}}+\frac{N(m-1)(\operatorname{diam} \Omega)^{m-1}}{A(T-t)^{(2 \sigma+l)(m-1)}} \\
-\frac{c\left(1+\frac{A}{2}\right)^{p}|\Omega|}{(T-t)^{l(p+q)}} .
\end{gathered}
$$

Case 2: If $r>A$, then $\omega(r) \leq 1, \quad \omega^{1-m}(r) \geq 1$, $\omega^{\prime}(r) \leq-1$, we have 


$$
\begin{aligned}
& v_{t}- \operatorname{div}\left(|\nabla v|^{m-2} \nabla v\right)-c v^{p}\left(x_{0}, t\right) \int_{\Omega} v^{q}(x, t) d x \\
&=\frac{m l-\sigma A}{m(T-t)^{l+1}}+\frac{N(m-1)(\operatorname{diam} \Omega)^{m-1}}{A(T-t)^{(2 \sigma+l)(m-1)}} .
\end{aligned}
$$

Since $p+q>m-1$, we can take $l>0$ such that $l p>l+1$. For such a fixed $l$, we choose a sufficiently small constant $\sigma$ such that $l+1>m l+2 \sigma$. Hence for sufficiently small $T>0$, setting $A>\max \left\{1, \frac{m l}{\sigma}\right]$, (7) and (8) imply that

$$
v_{t}-\operatorname{div}\left(|\nabla v|^{m-2} \nabla v\right)-c v^{p}\left(x_{0}, t\right) \int_{\Omega} v^{q}(x, t) d x \leq 0 .
$$

Let $\varphi(x)$ be a continuous positive function defined on $\bar{\Omega}$. Since $\varphi\left(x_{0}\right)>0$, there exist two positive constants $\rho$ and $\varepsilon$ such that $\varphi(x) \leq \varepsilon$ for all $x \in B\left(x_{0}, \rho\right) \subset \Omega$. Choose $T>0$ small enough to insure that $B\left(x_{0}, R T^{\sigma}\right) \subset B\left(x_{0}, \rho\right)$, hence we have $v(x, t) \leq 0$ on $\partial B\left(x_{0}, \rho\right) \times(0, T)$. Moreover, it follows from (6) that $v(x, 0) \leq K \varphi(x)$ for sufficiently large $K$. Since $u(x, t)$ is positive in $Q_{T}$, then there exists a positive constant $\eta$ such that $u(x, t) \geq \eta$ in $B\left(x_{0}, \rho\right) \times[0, T)$. Thus by using the comparison principle we have $v(x, t) \leq u(x, t)$ provided that $u_{0} \geq K \varphi(x)$. This shows that $u(x, t)$ blows up in a finite time.

Theorem 3.5 Assume that $u_{0}(x)$ and ${ }^{c}$ are large enough. Then the solution to (1) blows up in finite time provided that $p+q+1>m$.

Proof. Let $\varphi(x)$ be the unique positive classical solution to the linear elliptic problem

$$
\left\{\begin{array}{l}
-\operatorname{div}\left(|\nabla \varphi|^{m-2} \nabla \varphi\right)=\eta_{0}, x \in \Omega, \\
\varphi(x)=0, x \in \partial \Omega
\end{array}\right.
$$

Where $\eta_{0}$ is a positive constant.

Define a function $\bar{w}(x, t)$ as follows:

$$
\bar{w}(x, t)=\bar{v}(t) \varphi(x) .
$$

Where $v(t)$ is a solution for the following ordinary differential equation

$$
\vec{v}(t)=b \bar{v}^{p+q}(t), \quad \bar{v}(0)=\bar{v}_{0},
$$

where $b$ is a small constant enough, $0<\bar{v}_{0} \leq \min _{x \in[0, a]} u_{0}(x)$.

$$
\text { Let } \bar{A}=\max _{x \in \bar{\Omega}} \varphi(x), \quad \hat{A}=\min _{x \in \bar{\Omega}} \varphi(x) .
$$

Since $u_{0}(x)$ is large enough, setting $\bar{v}_{0} \bar{A} \leq \min _{x \in \bar{\Omega}} u_{0}(x)$, then we have

$$
\bar{w}(x, 0)=\bar{v}(0) \varphi(x) \leq \bar{v}(0) \leq \min _{x \in \bar{\Omega}} u_{0}(x) .
$$

On the other hand,

$$
\begin{aligned}
\bar{w}_{t}- & \operatorname{div}\left(|\nabla \bar{w}|^{m-2} \nabla \bar{w}\right)-c \bar{w}^{p}\left(x_{0}, t\right) \int_{\Omega} \bar{w}^{q}(x, t) d x \\
& =\bar{v}^{\prime}(t) \varphi+\eta_{0} \bar{v}^{m-1}-c \bar{v}^{p+q} \varphi^{p}\left(x_{0}\right) \int_{\Omega} \varphi^{q} d x \\
& \leq b \bar{v}^{p+q} \bar{A}+\eta_{0} \bar{v}^{m-1}-c|\Omega| \bar{v}^{p+q} \hat{A}^{p+q} \\
& \leq \bar{v}^{p+q}\left(b \bar{A}+\eta_{0}-c|\Omega| \hat{A}^{p+q}\right),
\end{aligned}
$$

here we use the condition $p+q>m-1$. Thus by using the comparison principle we have $\bar{w}(x, t) \leq u(x, t)$ provided that $c \geq \frac{b \bar{A}+\eta_{0}}{|\Omega| \hat{A}^{p+q}}$. It is easy to see that the solution of Problem (10) $\bar{v}(t)$ blows up in finite time. This shows that $\bar{w}(x, t)$ defined by (9) blows up in a finite time. Hence the solutons of Problem (1) $u(x, t)$ blows up in a finite time.

\section{ACKNOWLEDGMENT}

This work was financially supported by the Department of Education for Jilin Province (2013439), (2014195).

\section{REFERENCES}

[1] C. V. Pao, Asymptotic behavior of solutions of reaction-diffusion equations with nonlocal boundary conditions, J. Comput. Appl. Math. 88(1998), 225-238.

[2] A. Gladkow, K. Ik Kim, Blow-up of solutions for semilinear heat equation with nonlinear nonlocal boundary condition, J. Math. Anal. Appl. 338(2008), 264-273.

[3] Yunzhu Gao,Wenjie Gao, Existence and Blow-up of solutions for porous medium equation with nonlinear nonlocal boundary condition[J], Applicable Analysis. 90(5)(2011), 799-809.

[4] Yunzhu Gao, Wenjie Gao, Study of solutions to an initial and boundary value problem for certain systems with variable exponents Boundary Value Problems, doi:10.1186/1687-2770-2013-76.

[5] F. C. Li, C. H. Xie, Global and blow-up solutions to a p -Laplacian equation with nonlocal source, Comput. Math. Appl., 46(2003): 15251533.

[6] Z. G. Lin, Y. R. Liu, Uniform blowup profile for diffusion equations with nonlocal source and nonlocal boundary, Acta. Math. Sci., 24B(3)(2004): 443-450. 